\title{
Drowsiness Detection for Vehicle Drivers with Alert System
}

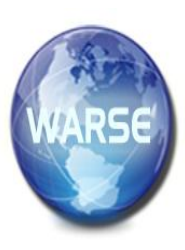

\author{
Vinamae M. Leopoldo ${ }^{1}$, Rodrigo S. Pangantihon $\mathrm{Jr}^{2}$ \\ ${ }^{1}$ University of Mindanao, College of Engineering Education, \\ Electronics Engineering Program, Matina, Davao City, 8100, PHILIPPINES \\ vinamaeleopoldo@umindanao.edu.ph \\ ${ }^{2}$ University of Mindanao, College of Engineering Education, \\ Computer Engineering Program, Matina, Davao City, 8100, PHILIPPINES \\ rodrigo_pangantihon@umindanao.edu.ph
}

\begin{abstract}
Being drowsy while driving is deemed a very dangerous thing. It is important to address that problem since drivers' lives are at risk. Preventing accidents would be too hard for them if they feel drowsy. This study aims to develop a device to help drivers, especially at night, to secure themselves from accidents due to drowsiness or sleepiness. It is especially sought to design an electronic device that will detect a person who is drowsy while driving and will trigger an alert system in terms of sound alarm and vibration in the pillow that leans on the driver's seat for the purpose of waking him up. Through Arduino Uno, a device was programmed and configured to detect drowsiness and activate an alarm as the output. On the other hand, an eye blink sensor was used to figure out if the person is drowsy or not. The result of the study showed that using an IR sensor as an eye blink sensor is feasible to determine the state of drowsiness of the driver. Upon testing, the system has achieved an accuracy rate of $80 \%$.
\end{abstract}

Key words : Drowsiness, Driving Risks, Eyeblink sensor, Alert system, Vibrator, Buzzer

\section{INTRODUCTION}

Sleepiness is a major cause of accidents on the road. It is much risky to travel at night or even in a day if the person is sleepy and it confers a higher probability to get into accidents [1]. Another study shows that sleeping while driving even in a short time could cause serious consequences [2]. In 2014, it is reported that $19.5 \%$ of the drivers had fallen asleep while driving and $15.9 \%$ having been close to falling asleep while driving. [3]. The latest studies had mentioned that in this world, there are $20-50 \%$ vehicle accidents because of fatigue [4]. The problem is about being drowsy while driving. Because of the dangers that drowsiness presents on the road, methods need to be developed for counteracting its effects.
There are studies that show that the changes and movements of the eye are the focus to detect the fatigue condition of a person [5]. The driver's blinking rate and eyelid movements when drowsy are different from their normal situations. The study shows that by measuring the number of eye blinks and the period time that the eyes are closed can figure out if the driver is drowsy [6]. Therefore, eye blinking is a basis to detect drowsiness. A person's attentiveness can be determined through its eye blink pattern. If a person is attentive, its eye blink pattern is different from those people who are sleepy or drowsy. Many methods have been proposed to automatically notice some eye blinks based on video series. A study uses image processing to monitor the fatigue and distraction of a driver [7]. Though there are devices that use image processing however it is highly costly [8]. A sensor that is not expensive but can be used in detecting eye blink is the eye blink sensor. Using an IR sensor, the infrared light will reflect into the surface of the eye detecting the eyelid movement. Eye Blink Sensor is safe, noninvasive for the eyes. It uses invisible light signals to pass in front of the naked eye [9].

The number of eye blinking of a person in a normal state varies from 12-19 per minute, thus eye blink frequency below this normal state denoted as drowsy [10]. Thus, normal eye blink interval if active is from 2 to 10 seconds [11]. Therefore, the eye blink interval above 10 seconds is not active. On the other hand, the NHTSA study proposed that $80 \%$ of collisions happen in just 3 seconds of distraction [12]. Moreover, another study shows that if a person's eyes are not focused on the road in just 2 seconds or longer, it could cause accidents [13]. Knowing these things would be helpful to know if a person is drowsy or not through the eye blink sensor. Another highlight of a study uses the invention of two vibrators as an alarm. Its vibrators are mounted in the vehicle. [14]. There is also a device used for waking up a driver and an operator has control element over time, and an alarm to produce vibrations in the control element [15]. It only shows that using a vibrator 
as an alarm is beneficial for waking up a person from sleeping. Another way of alerting a person is through a buzzer. The sound of a buzzer can wake up the driver from falling asleep. [16]. Using an available device which is the piezoelectric buzzer can be used as an alarm for it emits an audible alarm. [17].

Many researchers had been using an eye blink sensor to detect the driver's eye blink. Their purpose is to know if the driver is drowsy or not. If the driver is drowsy, they had used sound alarms. But sometimes, other people can't wake up in a sound alarm basis. That is why it needs a device that can wake up the driver using other senses. One thing that is effective to wake up a person is by using a vibrator that a driver may feel at his back.

This study aims to develop a device to help drivers, especially at night to secure their selves from accidents due to their drowsiness. It is especially sought to design an electronic device that will detect a person who is drowsy while driving and gives an alert system in terms of sound alarm and vibration of the pillow that leans on the driver's seat to wake him up.

Being drowsy while driving is a very dangerous thing. It is important to solve that problem since driver's lives are at risk. Preventing accidents would be too hard for them if they feel drowsy. To solve it, this device is designed to drivers who are traveling especially at night to feel safe and help them to alert the person from drowsiness if they did so.

This paper is focused on eye detection using an eye blink sensor. It is the one who senses if a person's eye blinking is in a drowsy state while driving. The eye blink sensor detects the blinking of the eye of a driver. The normal eye blink rate is 12-19 per minute, this indicates that the driver is not in a drowsy state and will not act to alarm. Eyeblink frequency below this normal state denoted as drowsy and the microcontroller will take an action to alarm. The normal blink interval is $2-10$ seconds. Therefore, above 10 seconds blink interval is in a drowsy state. This will trigger the microcontroller to send information to the buzzer and to the vibrators to function. The system can't only detect the number of blinks but also alarms if the eyes of the driver are closed within 2-3 seconds. Hence, the alarm system of the device will wake the person using his two senses - the sense of touch and sense of hearing. There will be alarm buzzer and vibrations of the driver's seat to alert the person from drowsiness. The device can only detect the frequency of the eye blinking and the number of seconds that the eye of a person is closed. It cannot detect the person's breathing or sigh, palpitations of the heart and the position of the body. Furthermore, its detection may only apply to the person who's wearing the eye blink sensor. Thus, the eye blink sensor is an IR sensor which means it can absorb light rays from the sun, so this device may only apply to those vehicles that are tinted.

\section{MATERIALS AND METHODS}

\subsection{Conceptual Framework}

The conceptual framework of the study is shown in figure 1 . The number of blinking eyes and closed eyes is the input to the system. The microcontroller will analyze, interpret and process if the person is in normal or drowsy state. If the person is in drowsy state, the buzzer and the vibrator will execute its intended functions.

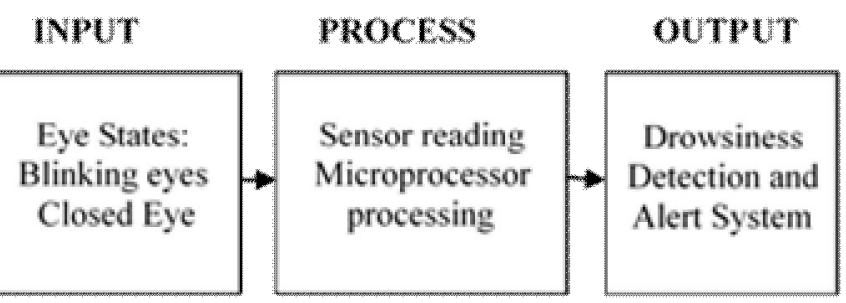

Figure 1: The Conceptual Framework

At the onset, there were two proposed designs to realize the device. One design has a wired connection from the eye blink sensor to the Arduino. The other one uses Bluetooth as a wireless connection from the eye blink sensor to the Arduino. In this case, a trade-off analysis has been done to discern which of the two designs is worthy to be implemented. The design which has a wired connection is being used in this study based on the result of the trade-off analysis using Pugh Matrix model.

\subsection{System Block Diagram}

As reflected in figure 2, eye blink sensor detects the blinking eyes and closed eyes. The microprocessor which is the Arduino will process if the collected data from the eye blink sensor are in a normal state or drowsy state. The $V_{D C}$ source is connected to the buzzer and the vibrator. The relay is connected between the Arduino and the alarm which is the vibrator and the buzzer. If the device needs to alarm, through a relay, the buzzer and the vibrator will take its course. The Power Transistor 2SD1213 was utilized as a relay to the vibrator and BJT (Bipolar Junction Transistor) pn100 as a relay to the buzzer.

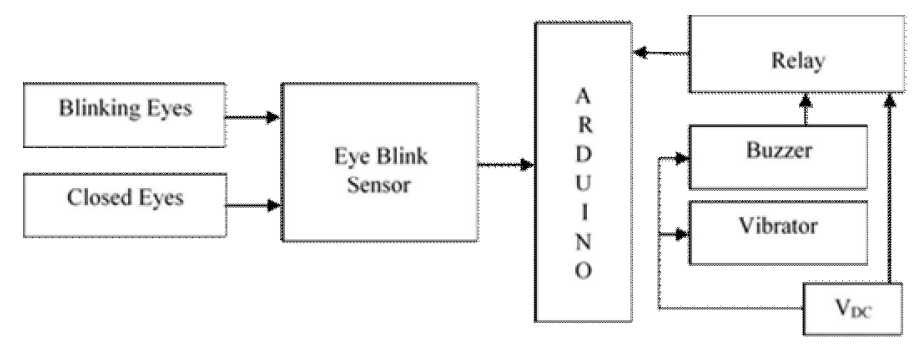

Figure 2: Functional Block Diagram 


\subsection{Hardware Development}

There has to be a fabricated box where the components are assembled. The components inside the box are the microcontroller, the relay, and the buzzer. The box is made of the acrylic board from high-quality cast acrylic achieving a classy finish. The size of the box is $4 \times 5 \times 2$ inches which can be placed at the side of the seat inside the vehicle. On the other hand, the IR sensor is attached to a safety glass which is $4 \times 6 \times 6$ inches. The photodiode and the phototransistor were removed from the circuit board of the sensor and were attached at the upper left corner of the safety glass. The tiny circuit board of a sensor is attached to the side of the glass. Lastly, the vibrator is attached inside the pillow. The size of the pillow is $2 \times 2 \mathrm{ft}$. Inside of it is a vibrator which is $37 \times 17 \times 11$ $\mathrm{cm}$.

The eye blink sensor has a big role in the whole device. The IR Reflective Sensor TCRT5000 was used as an eye blink sensor capable to detect the blinking of an eye. It has both a Photodiode and a Phototransistor coupled in its package. The photodiode can be used to generate an IR signal. The photodiode emits IR rays to the eye of the driver while the phototransistor is the receiver of the reflected signal from the eyeball. And because of this, the IR should be placed at the glass where the IR can reach the eyeball. The IR sensor is attached to the glass. The sensor can be triggered once there is a movement of an eye that counts as a blink. If the eye is open, there is no output from the eye blink sensor. But if the eye is closed, the eye blink sensor will give an output to the Arduino. A glass is fabricated based on a person who will use the eye blink sensor because the researcher wants to make sure that the IR signal is reflected in the eyeball.

Regarding the alert system, it needs to have a buzzer and a vibrator to alert the driver from drowsiness. PS1720P02 was used because it has a simple design with high efficiency in terms of performance. It has a very low power dissipation compared to electromagnetic components but has a high sound pressure. On the other hand, choosing a good vibrator would be very beneficial for alerting a person from drowsiness. This device had used the Vibroaction Slimming Belt. It is used to slim down specific body parts. But in this study, it has to be used as a vibrator to alert the driver. The vibrator is attached inside the pillow. The pillow will be placed in the driver's seat.

\subsection{Software Development}

Arduino Uno is a microcontroller that can be used to program a device. In this study, the Arduino is being programmed to determine if the driver is drowsy. The flow chart is shown in figure 3. First, the eye blink sensor will detect the number of eye blinks and the number of seconds that the eyes are closed. Through it, the microcontroller will analyze if the gathered eye blink detection is in the normal state or drowsy state. Normal State means the driver has 12-19 blinks per minute and 2-10 seconds blinking intervals. If this occurs, the
Arduino will just start the loop again and will not take any action. On the other hand, Drowsy State means that the driver's blinking rate is out of normal. If the driver doesn't blink in 10 seconds or the driver had closed his eyes for 2-3 seconds then the Arduino will send an output to the vibrator and the buzzer to function. Thus, if the blinking rate of the driver is less than 12 or more than 19 blinks per minute, then the Arduino will send triggering signal to the buzzer.

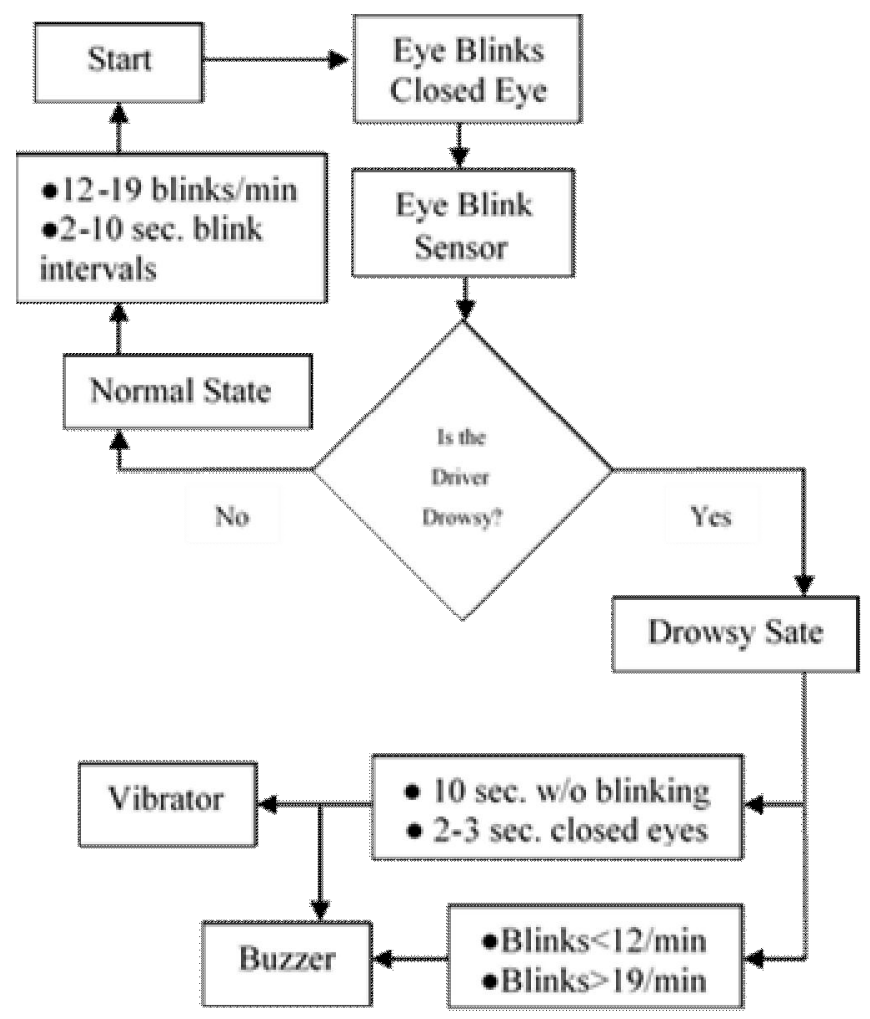

Figure 3: System Flowchart

\subsection{Control System}

The control system of the whole device is shown in figure 4. Through IR Reflective Sensor, the device can detect the number of eye blinks per minute and the number of seconds that the eyes are closed. The Arduino will process the gathered data of eye blinks and closed eyes. If the gathered data is in a normal state, then it will give feedback to the loop without taking any action. If the gathered data is in a drowsy state, the relay will switch the connections that will able to flow the current from the 12 Volt source to the Vibrator and the Buzzer.

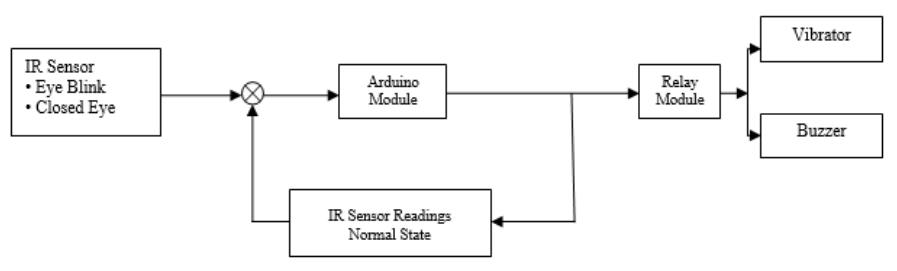

Figure 4: The Control System of the Device 


\subsection{Function Test Procedures}

The device needs to be tested to determine if it is working. The eye blink sensor should be calibrated first to achieve the best range of detecting eye blinks. To make sure that the data gathered from the eye blink sensor is accurate, it needs to test using the serial monitor of the Arduino Application. The serial monitor will display the number of blinks generated from the eye blink sensor and the number of seconds that the eyes are being closed. Thus, it could display if the eye blink sensor doesn't detect an eye blink within 10 seconds. It should be observed that every 10 seconds without blinking, the vibrator and buzzer should function. If the eye blink sensor detects that the eyes are closed for 2 seconds, then the buzzer and the vibrator should cause an alarm. Thus, if the number of blinks is less than 12 or greater than 19 per minute then the buzzer should alarm indicating that the driver is drowsy.

\section{RESULTS AND DISCUSSIONS}

\subsection{Schematic Diagram}

The schematic diagram of the device is shown in figure 5. The Arduino Uno is supplied with 12 Volt source. The IR Reflective Sensor is connected through its Vcc, GND, and the DO to the pin 2 of Arduino. The Vibrator is connected through the 12 Volt source to the Collector pin of the Power Transistor. Pin 12 is the output of Arduino connected to the Base pin of the Power Transistor to trigger as a relay to the vibrator. The Buzzer is connected from 12 Volt source to the Collector pin of the BJT, through it, if there's an output from pin13 in the Arduino to the base, the BJT will trigger as a relay to the buzzer. Both Emitter of the BJT and Power Transistor are connected to the ground.

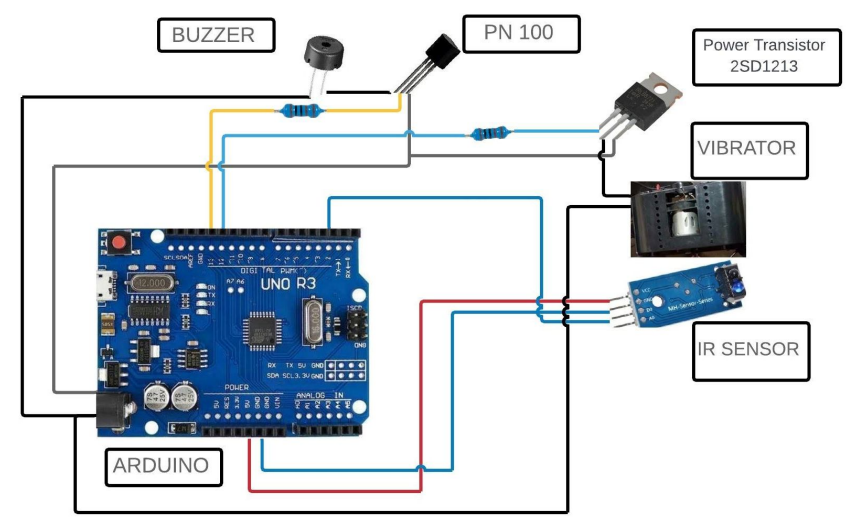

Figure 5: The final schematic diagram

\subsection{Final Prototype Design}

The final prototype design is illustrated in figure 6 . As seen in the figure, it is comprising of a glass, box, and a vibrator. The IR reflected sensor is attached to the upper right corner of the safety glass. The size of the glass is $4 \times 6 \times 6$ inches. Meanwhile, the Arduino, Bipolar Junction Transistor and Power Transistor are assembled inside the green colored box which is made of acrylic glass having the dimension of $4 \times 5 x$ 2 inches. The vibrator, in grey color, having a size of $37 \times 17 \times 11 \mathrm{~cm}$, is attached inside the $2 \times 2 \mathrm{ft}$ pillow placed at the back of the driver seat.

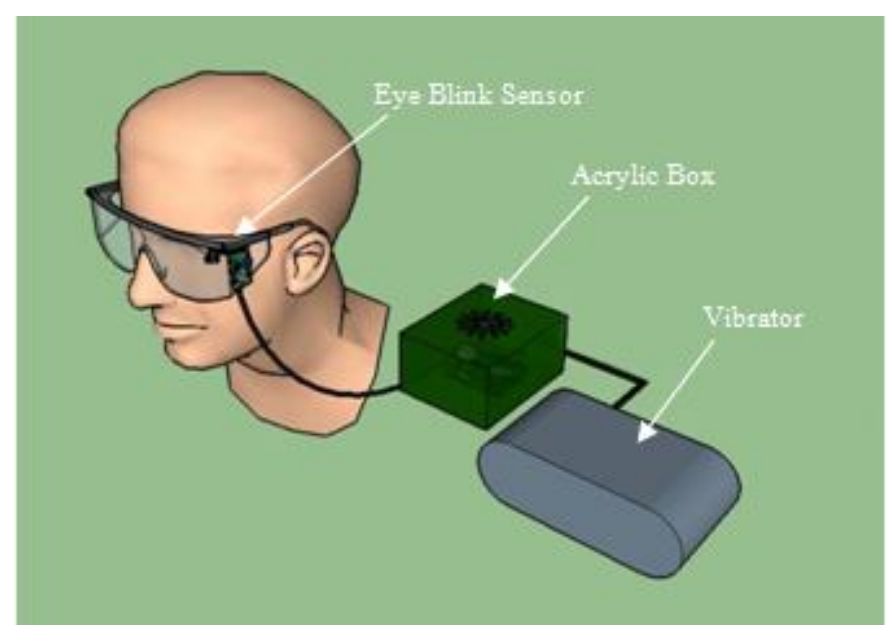

Figure 6: The final prototype design

\subsection{Functionality Tests}

To test the device in terms of its accuracy, one person-driver wore the device 10 times to know if the device does work. The driver is in a drowsy state if the driver's blinking is not in a normal range which is 12-19 blinks per minute. Thus, if the driver had closed his eyes for 2-3 seconds or his eyes are open for 10 seconds without blinking, it means the driver is in a drowsy state. In this case, the person pretends to be a drowsy driver. Based on the test, the driver's blinking is out of its normal range; the buzzer turns on at 10 out of 10 times being tested. The person also tests the device by letting the driver close his eyes for 2-3seconds, the vibrator and buzzer functions in 8 out of 10 times being tested. Lastly, the driver let his eyes blink for more than 19 blinks per minute, after a minute, the buzzer is on.

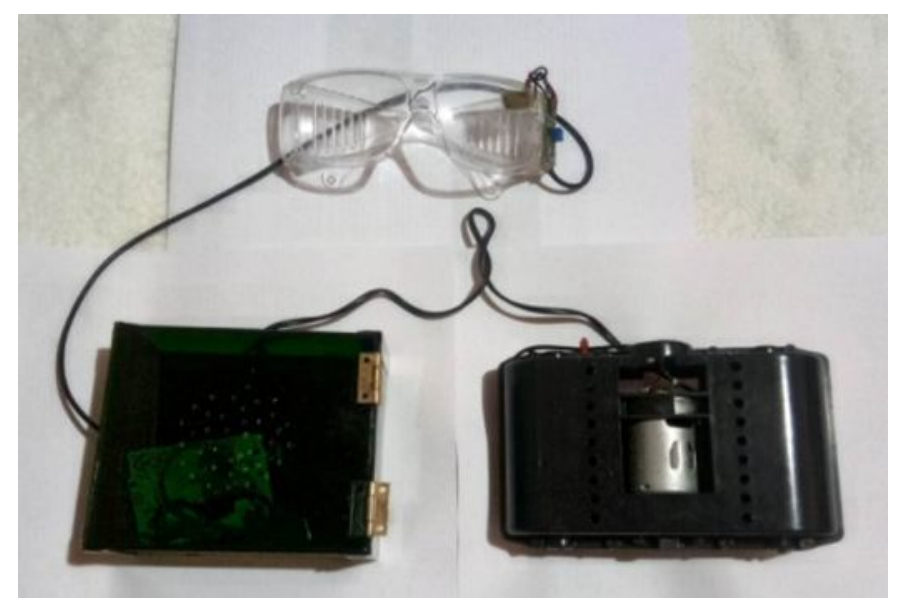

Figure 7: Actual Device 
One person also tests the normal state of the device. The normal state is when the driver is not drowsy indicating without any alarm. To know if it is normal, the driver's blinking must be within 12-19 blinks per minute. Thus, the blink interval has to be in between 2-10 seconds. As a couple

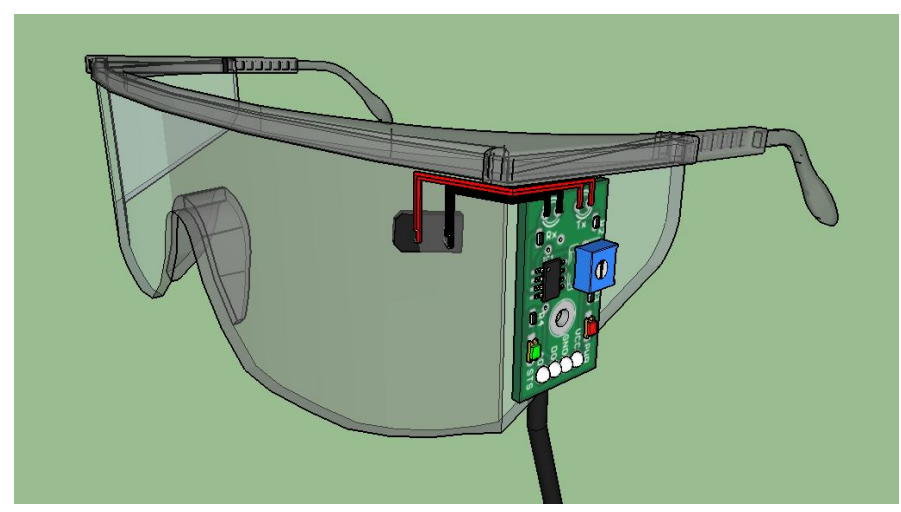

of minutes, in just normal blinking, the device did not take any actions indicating that the person is not drowsy. Another one had tested the normal state by 10 times, the device works at a normal state 8 times. See the actual device in figure 7 and eye blink sensor in figure 8 .

\section{Figure 8: Eye Blink Sensor}

\subsection{Accuracy of the Device Using Confusion Matrix}

To determine the performance of the device, one person has to undergo 20 testing trials. As reflected in Table 1, it is the confusion matrix recording different observations of the device [18]. It is predicted that in a normal state, there should be 20 normal states in the actual test and at drowsy state, there should be 20 drowsy states in the actual test. In the confusion matrix tool, the predicted normal state is referred to as predicted positive and the drowsy state as predicted negative. As for actual, the normal state is referred to the as true positive and drowsy state as true negative. Table 1 shows that out of 20 tests predicted to be at normal state, there are 17 times that the device had worked well. Whereas 3 times is predicted to be at normal state but in this case, the device reacts or gives a system response that the driver is on drowsy state even if it is not. System response means the device gives an alarm system which includes the vibrator and the buzzer to function. On the other hand, out of 20 tests predicted to be as drowsy state, there are 15 times that the device worked well and gives an alarm to the driver indicating that the driver is drowsy. Whereas 5 times predicted to be a drowsy state but, in this case, the device did not give a system response to the driver.

Table 1: Confusion Matrix

\begin{tabular}{lcc}
\hline & \multicolumn{2}{c}{ ACTUAL } \\
& Normal State & Drowsy State \\
\hline PREDICTED & & \\
Normal State & 17 & 3 \\
Drowsy State & 5 & 15 \\
\hline
\end{tabular}

As shown in Table 1, out of 20 times predicted to be at normal state, there are 17 times that the actual device worked well. And out of 20 times predicted to be at drowsy state, there are 15 times that the device worked well. The results of the confusion matrix in Table 1 show the values that could be used to calculate the sensitivity, specificity, accuracy, error rate and the precision of the device. The results have been calculated and are shown in Table 2.

Table 2: Measure of Values

\begin{tabular}{cc}
\hline Measure & Value \\
\hline Sensitivity & $77.27 \%$ \\
Specificity & $83.33 \%$ \\
Accuracy & $80.00 \%$ \\
Error Rate & $20.00 \%$ \\
Precision & $85.00 \%$ \\
\hline
\end{tabular}

\section{CONCLUSION AND FUTURE WORKS}

Based on the tests conducted, it clearly shows that using an IR sensor as an eye blink sensor is effective and feasible to determine if the person is in a drowsy state or not for as long as it is properly attached to a fix location of the glass. With the help of alarm modules consisting of the vibrator and the buzzer, the driver is being facilitated by the system to be awake while driving.

For future works, it is recommended to explore more sensors to detect the blinking rate because the IR sensor is sensitive and not durable for long time usage. On the other hand, regarding the connection between the eye blink sensor into the Arduino, it is recommended to use any device that will transmit the output of the eye blink sensor into a wireless connection as long as it cannot affect the delay process. In other fields, image processing may be used as many researchers are now into machine learning and neural networks.

\section{REFERENCES}

1. I. A. Akbar, T. Igasaki, N. Murayama, and Z. Hu, "Drowsiness Assessment using Electroencephalogram in Driving Simulator Environment," 2015 8th International Conference on BioMedical Engineering and Informatics (BMEI), 2015.

2. J. S. Higgins, J. Michael, R. Austin, T. Åkerstedt, H. P. A. Van Dongen, N. Watson, C. Czeisler, A. I. Pack and M. R. Rosekind, "Asleep at the Wheel-The Road to Addressing Drowsy Driving," Sleep., vol. 40, issue 2, Feb. 2017.

3. I. Radun, J. Radun, M. Wahde, C. N. Watling, and G. Kecklund, "Self-reported circumstances and consequences of driving while sleepy," Transportation Research Part F: Traffic Psychology and Behaviour., vol 32, pp. 91-100, July 2015.

4. J. Davidovic, D. Pešic' and B. Antic, "Professional drivers' fatigue as a problem of the modern era," 
Transportation Research Part F: Traffic Psychology and Behaviour, vol. 55, pp. 199-209, April 2018.

5. W. Tansakul and P. Tangamchit, "Fatigue Driver Detection System Using a Combination of Blinking Rate and Driving Inactivity," Journal of Automation and Control Engineering, vol. 4, no. 1, Feb. 2016.

6. V. Saini and R. Saini, "Driver Drowsiness Detection System and Techniques: A Review," International Journal of Computer Science and Information Technologies., vol. 5 (3), pp. 4245-4249, $2014 .$.

7. R. Jiménez-Moreno, S. A. Orjuela, P. Van Hese, F. A. Prieto, V. H. Grisales, W. Philips, "Video surveillance for monitoring driver's fatigue and distraction," Proc. SPIE 8436, Optics, Photonics, and Digital Technologies for Multimedia Applications II, 84360T, May 2012.

8. K. Thajee, P. Paengnakorn, W. Wongwilai and K. Grudpan,"Application of a webcam camera as a cost-effective sensor with image processing for dual electrochemical - colorimetric detection system," Talanta., vol 185, pp. 160-165, August 2018.

9. A. Frigerio, T. A. Hadlock, E. H. Murray, and J. T. Heaton, "Infrared-Based Blink-Detecting Glasses for Facial Pacing Toward a Bionic Blink," JAMA Facial Plastic Surgery, vol. 16, no. 3, pp. 211-218, April 2014.

10. R. Ahmad, and J. N. Borole, "Drowsy Driver Identification Using Eye Blink detection," International Journal of Computer Science and Information Technologies, vol. 6 (1), pp. 270-274, 2015.

11. A. Sahu, and S. R. Tandan, "Attention Analysis Of Candidates For Creating Virtual Interactive E-Classroom," International Journal of EngineeringResearch \& Technology (IJERT), vol. 2 issue 5 ISSN: 2278-0181, May 2013.

12. T. Mimar, "Driver distraction and drowsiness warning and sleepiness reduction for accident avoidance," U.S. Patent 9460601B2, Sept. 2009.

13. B.G. Simons-Morton, F. Guo, S.G. Klauer, J.P. Ehsani, A.K. Pradhan, "Keep your eyes on the road: Young driver crash risk increases according to duration of distraction," Journal of Adolescent Health, vol 54, issue 5, pp 561-567, May 2014.

14. J. G. Yurtz, "Automobile theft alarm," U.S. Patent 2885504A, May 1959.

15. K. Küntzel, "Device for waking up a driver and an operator," U.S. Patent 8810412B2, Aug. 2014.

16. (2017) Google Patents website. [Online]. Available: https://patents.google.com/patent/WO2018211583A1/e $\mathrm{n} ? \mathrm{q}=$ buzzer $\& \mathrm{q}=$ avoids $\& \mathrm{q}=$ driver $\& \mathrm{q}=$ falling+asleep\&be fore=priority:20171231\&after=priority:20170101\&oq= buzzer+avoids+the+driver+from+falling+asleep +2017

17. D. A. Warren, L. F. Heyl, E. H. Satterthwaite, Jr. S. Clark, A. W. Goldenson, D. Ho, N. U. Webb, J. Deluliis, T. Moore, B. Manuto, "Systems and methods for coordinating and administering self tests of smart home devices having audible outputs," U.S. Patent 10380878B2, May 2015.
18. R.J.A. Basan, K.T. Arizobal, N.C.T. Obas, and R.S. Pangantihon Jr. (2020). Development of Toilet Air Quality Assessment and Monitoring System. International Journal of Advanced Trends in Computer Science and Engineering, 9(4), 6821-6828. doi: 10.30534/ijatcse/2020/382942020 